\title{
Numerical investigation on the effect of specimen gripping arrangement on dynamic shear characterization using Torsion Split Hopkinson Bar
}

\author{
Bhaskar Ramagiri ${ }^{1 *}$, and Chandra Sekher Yerramalli ${ }^{1}$ \\ ${ }^{1}$ Department of Aerospace Engineering, IIT Bombay, India
}

\begin{abstract}
Torsion Split Hopkinson Bar (TSHB) is widely used in the dynamic shear characterization of material under pure shear loading. In TSHB, tubular specimens with either circular or hexagonal flanges are used. The specimens with circular flanges are generally bonded using adhesive to the incident and transmission bars. The specimens with hexagonal flanges are gripped into the hexagonal holders that are fixed onto incident and transmission bars. In the current study, numerical simulations are carried out to see the effect of gripping arrangements on the dynamic shear characterization quality. Numerical experiments with three gripping configurations are studied - the first gripping configuration with a direct bond (numerically-tie) between specimen and bars. The second configuration with the specimen gripped by hexagonal holders fixed to bars. The third configuration with specimen directly gripped into the incident and transmission bars having hexagonal slots.
\end{abstract}

\section{Introduction}

Torsion Hopkinson Bar (TSHB) is used for determining the shear response of material for strain rates in range of $10^{2} \mathrm{~s}-1$ to $10^{4} \mathrm{~s}-1$ [1]. Tubular specimens are proven to be effective in dynamic shear characterization[2]-[4]. In TSHB, the specimen needs to be firmly gripped without any slip in for characterization. Adhesive bonding of tubular specimen between incident and transmission bar is commonly used technique in TSHB[5]. The bonded specimen in TSHB is shown in Figure.1. This technique requires to wait till the bond is cured for carrying out experiment. The bond needs to be as thin as possible to avoid the wave reflections at the bonding zone. When testing material with high stiffness and strength, the adhesive shear strength needs to be sufficient enough to avoid failure at the bonded region. However, even when large diameter circular flanges are used, the limited strength of the adhesives allow testing merely low strength materials. To overcome these disadvantages in adhesive bonding, the specimens are gripped using hexagonal holders. The hexagonal holders have to be designed and machined with care to tightly fit the specimen and avoid slip. The holders are fixed to incident and transmission bars by using threaded bolts as shown in

* Corresponding author: bhaskar.r@aero.iitb.ac.in 
Figure.2. The other technique to grip the specimen is to directly fit the specimen between incident and transmission bars by making hexagonal slots on the bars as shown in Figure.3. Numerical experiments are carried out on these three specimens gripping configurations to access the quality of shear characterization. The simulations are carried out in ABAQUS/Explicit academic research software.

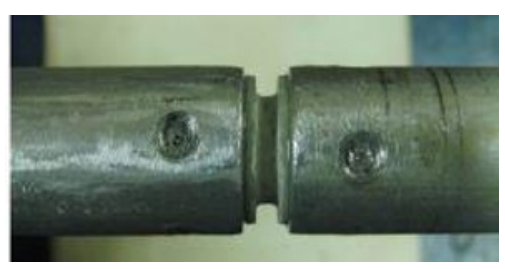

Fig. 1. Adhesive bonded tubular specimen.

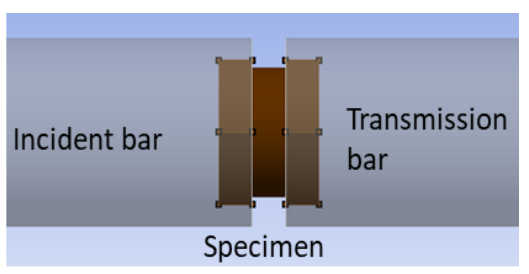

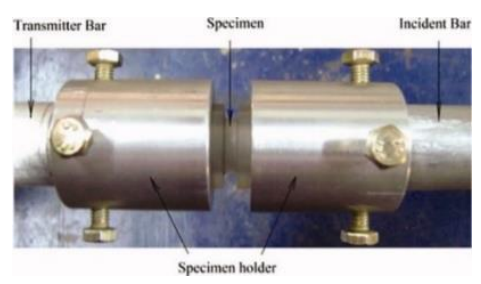

Fig. 2. Specimen gripped by hexagonal holders.

Fig. 3. Specimen gripped in hexagonal slots on bars.

\section{Finite element modelling}

The three gripping cases are simulated with same incident and transmission bar lengths. The incident bar is of $1.5 \mathrm{~m}$ and transmission bar is of $1 \mathrm{~m}$. The incident, and transmitter bars are tubular with outer diameter of $25.4 \mathrm{~mm}$ and inner diameter of $8 \mathrm{~mm}$. The same specimen geometry is used for all the three gripping cases. The gauge length of the specimen is $5 \mathrm{~mm}$, the internal diameter is $12 \mathrm{~mm}$ and the thickness of the specimen is $0.5 \mathrm{~mm}$. In case-1(the perfectly bonded case), the specimen and the bars are bonded using tie interaction. In case2(holder with hexagonal slot case), the holders are tied to the bars and the specimen surface and grip surface are given with frictional interaction. In case 3, the specimen surface and the grip surface are given with frictional contact. In case 2 and case 3, the specimen geometry is designed to exactly fit into grips without any slip. In Torsion Hopkinson bar, generally a portion of the incident bar is clamped and loaded in torsion to develop stored torque. The stored torque is then released spontaneously by breaking a fracture bolt to produce incident pulse. The length of clamped section and the rotational displacement determines the pulse duration and amplitude of the incident pulse. In the current work, to reduce the numerical problem size the incident pulse is generated by using angular velocity pulse to the free end of the incident bar. The amplitude of the angular velocity is set in a way that the bar does not cross the yield limit. The amplitude of the angular velocity is calculated using the equation 1.

$$
\dot{\theta}=\frac{T}{Z_{b}}=\frac{\tau_{y i e l d^{*}} J_{b a r}}{r_{b a r^{*}} Z_{b a r}}
$$

Where, $Z_{\text {bar }}$ is torsional Impedance of the bar.

Elastic Plastic material data is used for both the specimen and bars. The material data curve used in the present numerical work is shown in Fig.4. 
Table 1: Material properties AA6061-T6

\begin{tabular}{|l|r|}
\hline Density r (kg/m3) & 2700 \\
\hline Young's modulus E $(\mathrm{GPa})$ & 70 \\
\hline Poisson's ratio n & 0.3 \\
\hline
\end{tabular}

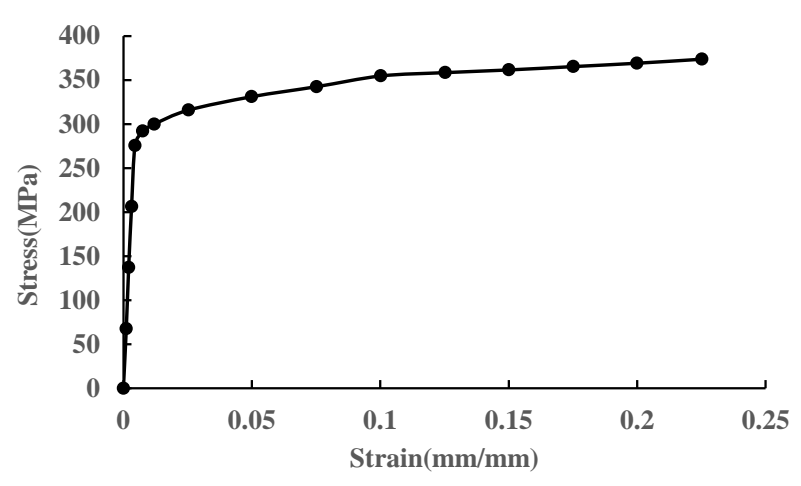

Fig. 4. Material data used for both bar and specimen

The bars and specimen are meshed using C3D8R elements (8-node linear brick, reduced integration, hourglass control). The mesh size of the bars is $1 \mathrm{~mm}$ and mesh size of the specimen is chosen to have four elements through the thickness in gauge section of the specimen. The meshed model is presented in Fig.5. The meshed specimen is shown in Fig.6.

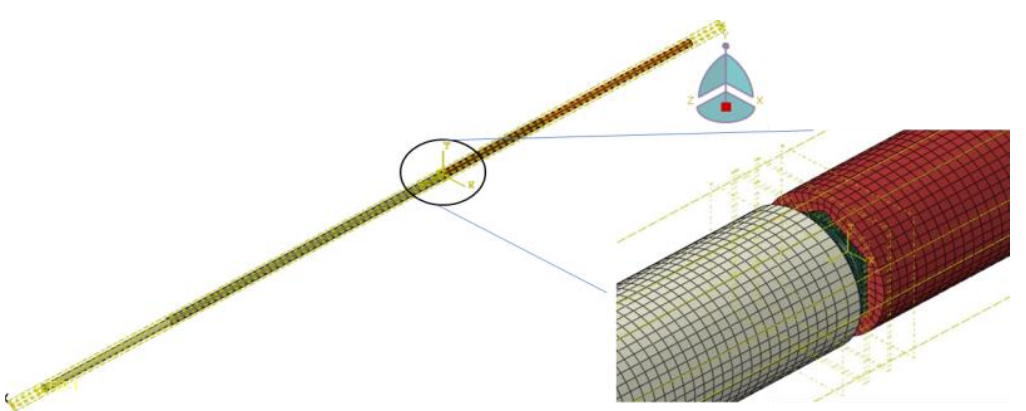

Fig. 5. Meshed model of the TSHB

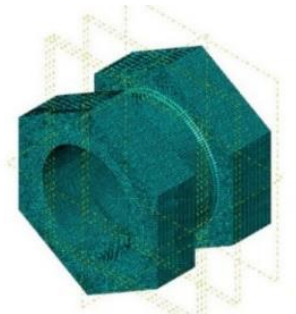

Fig. 6. Meshed Specimen using C3D8R elements 
The Tie constraint used for holding the specimen is checked for its effectiveness in perfectly bonding the specimen by carrying out numerical calibration study. The incident and transmission bar surfaces are directly bonded using tie constraint and an incident angular velocity pulse is given as input. The simulation output of incident pulse taken from an element on the incident bar and transmission pulse from an element on the transmission bar are found to be equal and is plotted in Fig.7.

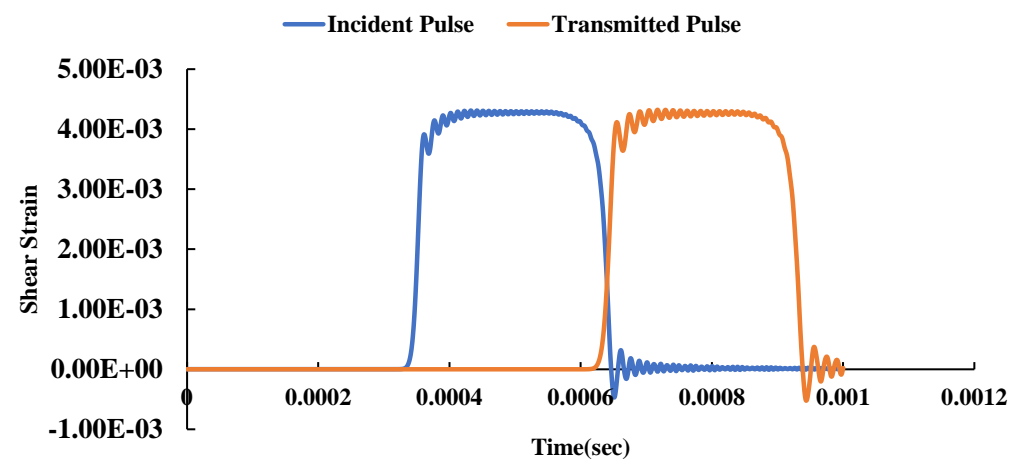

Fig.7. Calibration to find the effectiveness of Tie constraint

\section{Results}

\subsection{Perfectly bonded with hexagonal flanges specimen (case-1):}

In perfectly bonded case, the specimen end faces are directly tied to incident and transmission bar interfaces. The incident, reflection and transmission pulses are taken using element shear strain data. The solution is solved in Cartesian coordinate system. The solution is then transformed to cylindrical coordinate system using user defined transformation tool in visualization of ABAQUS/Explicit. The specimen shear stress and shear strain of all the elements in the gauge section are directly taken from are averaged to compute the direct taken specimen shear stress- shear strain data. This data is compared with the TSHB formulation shear stress-shear strain plot.

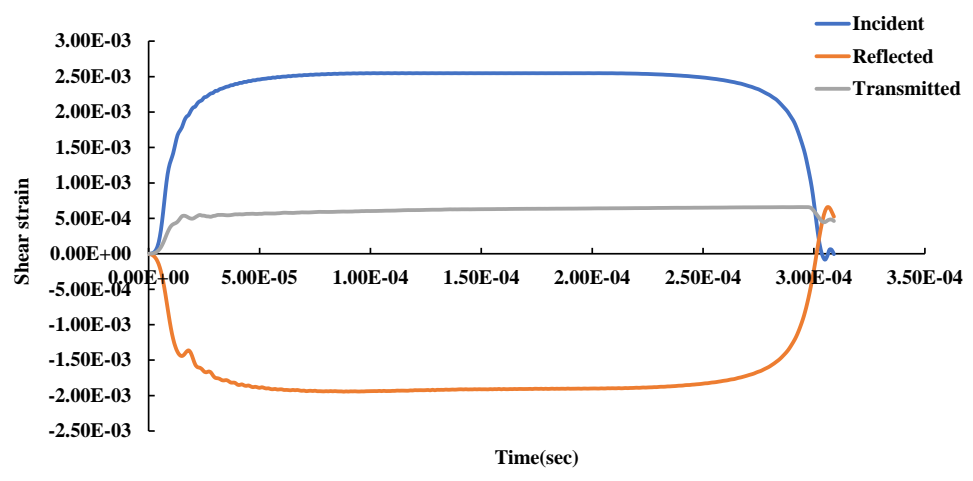

Fig. 8. Time synchronized shear strain pulses

The shear stress and shear strain using TSHB formulation is computed using the below formulation.

Shear stress:

$$
\tau_{\text {specimen }}=\frac{G_{b} J_{b}}{2 \pi r_{s}^{2} t_{s} r_{b}} \gamma_{\text {transmitted }}(t)
$$


Shear Strain:

$$
\gamma_{\text {specimen }}=\frac{2 C_{b} r_{s}}{r_{b} l_{s}} \int_{0}^{t} \gamma_{\text {reflected }}(t)
$$

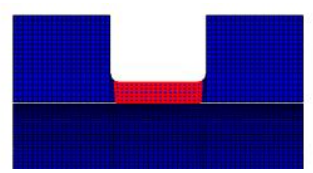

Fig. 9. Specimen elements for computing direct shear stress-shear strain

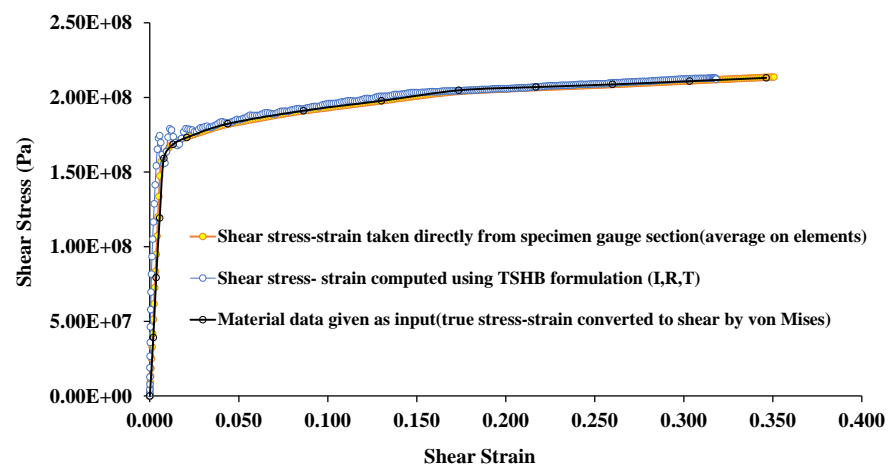

Fig. 10. Perfectly bonded case shear stress-strain comparison

\subsection{Specimen gripped in slot on bars (case-2)}

The computed shear stress- shear strain from TSHB formulation using strain data taken from incident and transmission bar and the direct measurement from specimen are presented in Fig.11. The specimen shear stress-shear strain from perfect bond and specimen held in slot are in complete alignment. The slight disturbance which can be neglected is coming from contact interaction. The strain pulses from the incident bar and transmission bar from slot gripping case is also compared with other two cases in next section.

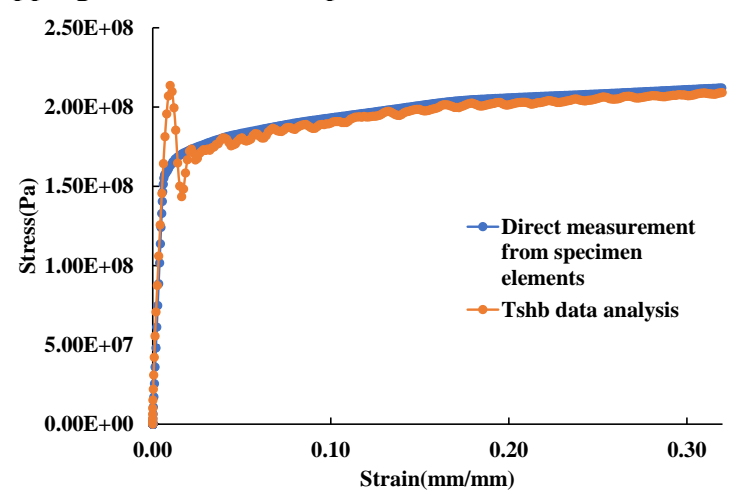

Fig. 11. Specimen in slot case shear stress-strain comparison 


\subsection{Specimen gripped in Holders on bars (case-3)}

The specimen stress computed from TSHB analysis using strain pulses from incident and transmission bar is compared with specimen shear stress- shear strain directly taken from the specimen in Fig.12. The difference in shear stress- shear strain data from TSHB analysis and the direct measurement is coming from the impedance $(\rho J C)$ mismatch brought in to system by holder geometry. This impedance difference crated is clearly visible from the incident and transmission bar shear strain data. The comparison of shear stress- shear strain data from all the three cases using TSHB analysis is presented in Fig.13.

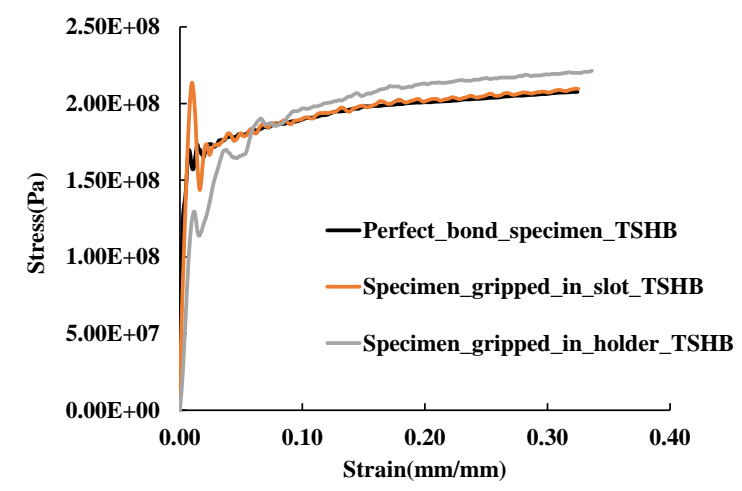

Fig. 12. Comparison of shear stress-strain from three cases using TSHB analysis

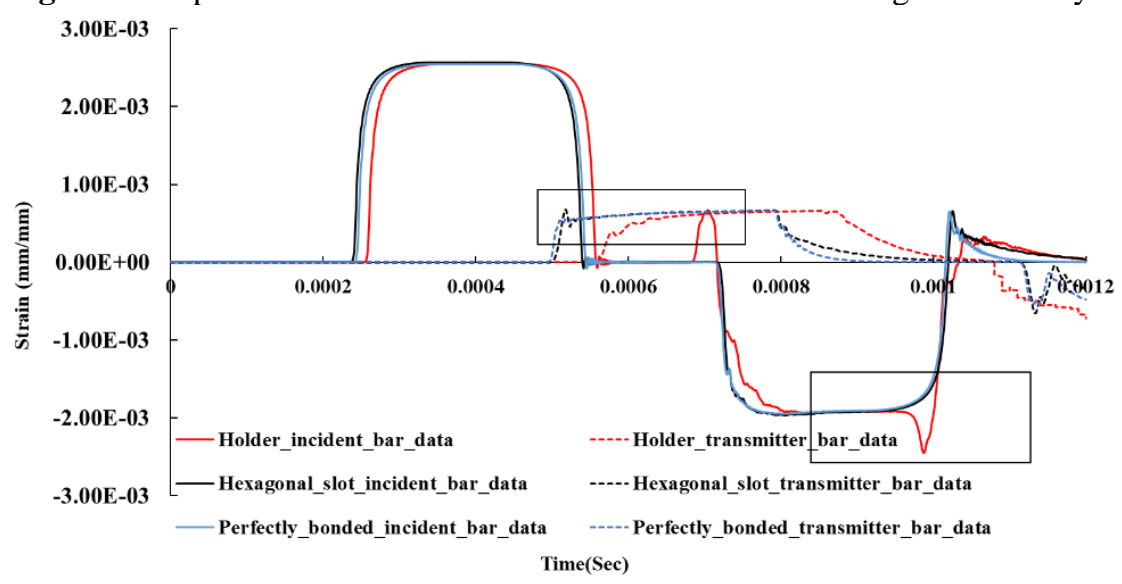

Fig.13. Comparison of Incident and transmission bar data from three cases

\section{Conclusions}

Numerical experiments have been carried out using three gripping configurations. The perfectly bonded case gives a more accurate way to capture the material shear response. The limited shear strength of the adhesive limits in characterizing strong material. This can be solved by using slots on the bars and external hexagonal holders. The numerical simulations show that having slots on the bars gives a better match of impedance and is able to capture the response accurately and is in good agreement with shear stress- strain data from bonded configuration. When using hexagonal slots care has to be taken to keep the impedance of the holder close to bar impedance to avoid discrepancies in capturing the shear response of the material. 


\section{References}

1. Y. Hu and R. Feng, "On the Use of a Kolsky Torsion Bar to Study the Transient Large-Strain Response of Polymer Melts at High Shear Rates," J. Appl. Mech., vol. 71, no. 4, p. 441, 2004, doi: 10.1115/1.1756142.

2. C. S. White, "Use of the Thin-Walled Torsion Specimen," p. 28.

3. W. F. Hosford, "Mechanical Behavior of Materials," p. 447.

4. C. Gang, H. Xicheng, C. Junhong, and Z. Weizhou, "Numerical Simulation on the specimen dynamic plastic deformation behaviour in the torsional split Hopkinson bar test," EPJ Web Conf., vol. 183, p. 01020, 2018, doi: 10.1051/epjconf/201818301020.

5. N. K. Naik, A. Asmelash, V. R. Kavala, and V. Ch, "Interlaminar shear properties of polymer matrix composites: Strain rate effect," Mech. Mater., vol. 39, no. 12, pp. 10431052, Dec. 2007, doi: 10.1016/j.mechmat.2007.05.003. 